Meta

Journal des traducteurs

Translators' Journal

\title{
La traduction phraséologique
}

\section{Claudia Maria Xatara}

Volume 47, numéro 3, septembre 2002

URI : https://id.erudit.org/iderudit/008029ar

DOI : https://doi.org/10.7202/008029ar

Aller au sommaire du numéro

Éditeur(s)

Les Presses de l'Université de Montréal

ISSN

0026-0452 (imprimé)

1492-1421 (numérique)

Découvrir la revue

Citer cette note

Xatara, C. M. (2002). La traduction phraséologique. Meta, 47(3), 441-444.

https://doi.org/10.7202/008029ar

\section{Résumé de l'article}

Presque tous les textes présentent quelque lexie complexe de la phraséologie des langues de spécialité ou de la phraséologie de la langue commune. Au traducteur incombe le devoir tout d'abord d'identifier ce phraséologisme, ensuite d'en saisir le sens. Il n'est pas suffisant, cependant, d'en proposer une explication dans la langue cible : le traducteur doit chercher à établir son équivalence phraséologique, c'est-à-dire qu'il doit trouver une unité lexicale correspondante pas seulement quant au sens, mais aussi quant à la formulation phraséologique.
Ce document est protégé par la loi sur le droit d'auteur. L'utilisation des services d’Érudit (y compris la reproduction) est assujettie à sa politique d'utilisation que vous pouvez consulter en ligne.

https://apropos.erudit.org/fr/usagers/politique-dutilisation/ 


\section{BLOC-NOTES}

\section{La traduction phraséologique}

\begin{abstract}
RÉSUMÉ
Presque tous les textes présentent quelque lexie complexe de la phraséologie des langues de spécialité ou de la phraséologie de la langue commune. Au traducteur incombe le devoir tout d'abord d'identifier ce phraséologisme, ensuite d'en saisir le sens. Il n'est pas suffisant, cependant, d'en proposer une explication dans la langue cible: le traducteur doit chercher à établir son équivalence phraséologique, c'est-à-dire qu'il doit trouver une unité lexicale correspondante pas seulement quant au sens, mais aussi quant à la formulation phraséologique.
\end{abstract}

\begin{abstract}
Almost all texts contain some complex lexical units belonging to the phraseology of the language of a specialized field or of the general language. The translator must first identify this phraseologism, and then understand its meaning. However, it is not enough to propose an explanation in the target language: the translator has to establish its phraseologically equivalent lexical unit in meaning and in phraseological formulation.
\end{abstract}

\section{MOTS-CLÉS/KEYWORDS}

idiomatismes, langues de spécialité, lexies complexes, traduction phraséologique

Ferdinand de Saussure (1969) fut le premier à attirer notre attention sur l'existence de combinaisons non libres, mais les particularités de ces combinaisons commencèrent à être développées surtout par Charles Bally (1951), quand il institua la phraséologie comme une discipline de la lexicologie. Ce sous-domaine se divise, à la fois, en phraséologie proprement dite, qui analyse et classifie tout le matériel disponible comme phraséologique, et la phraséographie, qui s'occupe de l'élaboration de méthodes théoriques et pratiques pour la confection de dictionnaires phraséologiques, recueils de ces combinaisons. Nous devons en plus séparer la phraséologie populaire et la phraséologie technique et scientifique.

Les principaux successeurs de Bally, quant à l'approfondissement des aspects théoriques de cette question, sont les Russes, comme Vinogradov, beaucoup plus que les linguistes occidentaux, parmi lesquels on trouve cependant comme pionniers, les Allemands Thun et Häusermann et le Nord-Américain Weinrich (Ettinger 1982, Tristá 1988). En s'appuyant sur les travaux russes, on a étudié les principes de disposition et de lecture du matériel lexicographique et l'inclusion de ces unités phraséologiques; les critères de sélection, de distribution et de définition des phraséologismes; l'analyse et la classification de l'amas phraséologique compris dans les dictionnaires généraux; le procédé d'archaïsation et de représentation des unités phraséologiques synonymes et leurs variantes; ainsi que les caractéristiques stylistiques des phraséologismes (Carneado Moré 1985).

L'étude de cette branche de la linguistique prend une importance de plus en plus grande, autant du point de vue théorique, dans l'investigation des règles lexicales, sémantiques et grammaticales, que du point de vue pratique, dans l'enseignement et l'apprentissage des langues nationales et étrangères, dans l'élaboration de dictionnaires, etc. C'est elle, donc, que nous avons mise en relief dans nos recherches, toujours en considérant la comparaison de la phraséologie française à la phraséologie portugaise (Xatara 1998).

Les combinaisons figées, nécessaires pour que l'expressivité de la langue se manifeste, sont des ensembles de mots qui n'ont presque pas de liberté de combinaison, mais qui sont stables (leur signifié est consacré, c'est-à-dire qu'il est reconnu par la communauté des sujets parlants), et qui ont des caractéristiques propres mais sont de nature diverse, chacune ayant une dénomination spécifique.

Les distinctions et les limites parmi plusieurs types de combinaisons figées, loin de donner lieu à un consensus, ne représentent que de simples tendances. On peut citer, comme unités phraséologiques de la langue générale: les clichés, les combinaisons conventionnelles à sens dénotatif, les expressions stéréotypées, les lieux-communs, les expressions idiomatiques, les phrases toutes faites, l'argot, les injures, les gros mots, les mots ou expressions scatologiques. Beaucoup d'autres combinaisons non libres: aphorismes, citations, dictons, maximes, proverbes et toutes les formulations figées et consacrées, révélatrices de l'âme des peuples, sont traitées par la parémiologie, un sous-domaine de la phraséologie. Comme unités phraséologiques de la langue de spécialité, on peut trouver les syntagmes terminologiques ou technico-scientifiques, les jargons et les argots professionnels.

L'acquisition de la plupart de ces unités phraséologiques, en langue maternelle, est réalisée de façon asystématique, à l'occasion des lectures ou conversations, si le locuteur se rend compte qu'il s'agit d'expressions consacrées. À ce moment, il pourra les mémoriser et les employer lorsque la situation et le contexte les transforment en un 
facteur spécifique d'efficacité communicationnelle. À cause de cela, il ne peut être valable pédagogiquement d'apprendre aux étudiants d'une seconde langue à déchiffrer des messages plus ou moins cultivés et à exclure des relations de complicité communes qui sont nourries par les natifs entre eux, ambiance où se placent les phraséologismes. Il n'existe pas d'individu qui, en conversation avec des étrangers dont il pense maîtriser la langue, ne considère très traumatisant de perdre la face dans ces situations banales de la vie quotidienne, quand il tombe sur un phraséologisme qu'il n'arrive pas à décoder (Galisson 1988). Faciliter, donc, pour les sujets parlants ou apprenants d'une seconde langue, l'incorporation de ces unités linguistiques conventionnelles, c'est la tâche d'un enseignement qui, aidé surtout par des dictionnaires spéciaux bilingues, met en valeur le lexique comme l'un des responsables de la fluidité et l'efficience de la communication souhaitée (Tagnin 1988).

Quant au traducteur, il doit préciser des mots qui désignent le mieux, dans la langue cible, la notion présentée dans le texte de la langue source. En fait, outre l'obligation de connaître presque toute la grammaire et une tranche assez raisonnable du lexique commun d'une langue, il est fondamental que le traducteur puisse se servir d'un grand répertoire de formes figées, en discernant leur signifié connotatif et en les rendant appropriés à des contextes spécifiques. À cette fin, il doit tout d'abord reconnaître l'unité de traduction minimale, qui est un problème de segmentation. Chaque lexie complexe, abondamment présente dans le discours familier, quand il s'agit des unités phraséologiques de la langue générale, et dans le discours technico-scientifique, dans le cas des unités terminologiques, représente une unité de traduction minimale, car en étant une lexie, quoique complexe, elle est par conséquent, la moindre unité de fonctionnement syntaxique (Molinie 1986).

Ayant identifié l'une de ces lexies comme unité de traduction minimale, le traducteur doit alors, quand il ne trouve pas dans la langue cible un équivalent satisfaisant à quelque phraséologisme du texte de la langue source, analyser la valeur de l'équivalent proposé par les dictionnaires, puisque le degré de fréquence et le registre relativisent même la portée des traductions. Par exemple, le traducteur devra reconnaître "finir en queue de poisson", comme unité de traduction minimale, et pas seulement «queue de poisson", pour la traduction phraséologique acabar em pizza. Mais le plus grand problème c'est que, dans la plupart des dictionnaires bilingues, ces unités lexicales, si elles sont répertoriées, ne sont que définies qu'avec les mêmes paraphrases présentées par les monolingues, au lieu d'être traduites, dans une correspondance appropriée avec l'autre langue en question, en précisant les conditions d'emploi.

Ainsi, en prenant en compte le français et le portugais, langues avec lesquelles nous travaillons, on a, pour «faire feu de tout bois» ou pour «s'embarquer sans biscuit», les paraphrases définitionnelles respectives utilizar todos os meios de que se dispõe no momento et meter-se num negócio sem dispor dos meios necessários para se sair bem dele, au lieu des traductions idiomatiques queimar todos os cartuchos et embarcar em canoa furada.

Le traducteur qui doit "ménager la chèvre et le chou", ne devra pas seulement administrar interesses contraditórios, mais il devra, idiomatiquement, acender uma vela a Deus e outra ao Diabo, ou alors agradar a gregos e troianos, ou encore jogar com pau de dois bicos. De la même façon, pour traduire les proverbes "Au royaume des aveugles, les borgnes sont rois» et " $\overline{\mathrm{A}}$ brebis tondue, Dieu mesure le vent», par exemple, il ne peut pas se satisfaire, respectivement, de l'explication «un médiocre quelconque paraît notable s'il est comparé à des gens sans aucune valeur» ou «Dieu donne les épreuves proportionnelles à la faiblesse humaine»; le traducteur ne doit pas non plus se contenter de la traduction littérale No reino dos cegos, os caolhos são reis pour le premier proverbe, ou Para ovelha tosquiada, Deus mede o vento pour le deuxième, mais il a à trouver les proverbes équivalents - figés et consacrés: Em terra de cegos, quem tem um olho é rei et Deus dá o frio conforme o cobertor.

Il diminuera aussi la qualité de la traduction si, par exemple, "glisser la peau de banane» reçoit en portugais une 'explication' comme enganar, lograr, burlar ou ludibriar, au lieu de son équivalence idiomatique précise, puxar o tapete ou dar uma rasteira; ou encore, levar vantagem ne doit pas être consideré comme la 'traduction' de l'idiomatisme "couper l'herbe sous les pieds", parce que, dans ce cas, la traduction 'idiomatique' convenable est passar a perna.

Les dictionnaires spéciaux devraient rendre mieux compte de la question en décrivant les unités lexicales sélectionnées par leurs caractéristiques spécifiques, par exemple, celles qui représentent les faux amis pour une autre langue, les verbes qui régissent des prépositions déterminées, les mots qui constituent des champs lexicaux du vocabulaire érotique ou les expressions considérées comme idiomatiques, tous ces renseignements pourraient faire l'objet d'ouvrages lexicographiques spéciaux. Dans les dictionnaires d'idiomatismes, on pourrait ainsi trouver avec une plus grande précision tirar água do joelho pour «changer l'eau des olives», ou rodar a baiana pour «jeter son bonnet par-dessus les moulins", ou encore chover no molhado pour «faire double emploi». 
S'il s'agit d'une lexie terminologique, la préoccupation est la même: il ne suffit pas de définir, bien que très correctement, son signifié; le traducteur doit présenter pour le mot de la langue source un équivalent terminologique dans la langue cible; ainsi pour "cour d'assises" il aura supremo tribunal federal, pour "appareil de détente», válvula redutora de pressão, pour "poutre maîtresse», viga mestra.

Seulement dans les cas où le phraséologisme d'une culture n'a pas de corrrespondant dans l'autre langue, il est conseillé de recourir à des gloses définitionnelles (Rézeau 1990): à une unité phraséologique peut correspondre, dans une autre langue, une formulation identique, ou une formulation pareille ou même une formulation assez différente, qui rende compte de visions du monde divergentes ou non, en respectant les spécificités culturelle, sociale et linguistique de chaque peuple.

Une bonne traduction phraséologique, bien que beaucoup de traducteurs considèrent les phraséologismes intraduisibles, doit prendre en compte non seulement la signification, mais aussi la situation de communication, ce qui inclut la différence entre l'oral et l'écrit et le degré d'ajustement de l'émetteur au récepteur, en plus de considérations d'ordre sociolinguistique.

La traduction appropriée, c'est-à-dire celle dont le sens est le plus semblable possible, pour le portugais brésilien de n'importe quelle unité phraséologique en français pourra être littérale ou non littérale. Nous n'employons pas ici, cependant, le sens rigoureux du mot "littéral»: on sait que rien ne sera jamais rigoureusement littéral en traduction, puisque l'on peut avoir de la littéralité quant à la forme, mais jamais quant à la signification.

La traduction littérale, beaucoup moins fréquente, qui a lieu quand le phraséologisme de la langue d'origine se concrétise dans la langue cible en unités identiques, aura les caractéristiques suivantes: présence d'équivalents lexicaux et conservation de la même structure (classe grammaticale et ordre syntagmatique), de même effet et de même niveau de langue. Si l'on cherche la correspondance, par exemple, d'expressions idiomatiques comme "arriver comme un ouragan", "fort comme un taureau », "faire comme le renard et les raisins", on trouve sans effort les équivalences chegar como um furacão, forte como um touro, fazer como a raposa e as uvas.

Les idiomatismes traduits de façon non littérale sont beaucoup plus nombreux et le mécanisme de traduction correspond en fait à trois types:

a) quand les phraséologismes se traduisent par des idiomatismes semblables aussi dans la forme $\Rightarrow$ absence d'équivalences lexicales totales, mais sans altération de structure, d'effet ou de niveau de langue: mouillé comme un canard $\rightarrow$ molhado como um pinto; promettre monts et merveilles $\rightarrow$ prometer mundos $\mathrm{e}$ fundos; travail arabe $\rightarrow$ seviço porco;

b) quand les phraséologismes se traduisent par des unités à forme bien diverse $\Rightarrow$ absence d'équivalences lexicales totales et altération de structure, d'effet ou de niveau de langue: avoir plusieurs cordes à son arc $\rightarrow$ ter muitas cartas na manga; entrer comme dans un moulin $\rightarrow$ estar como na casa da mãe Joana; tarte à la crème $\rightarrow$ carne de vaca;

c) quand les phraséologismes se traduisent par des paraphrases $\Rightarrow$ absence d'équivalences lexicales, cas où l'on fait appel à des gloses recours fréquent entre les cultures assez différentes (Rey 1986) — ou des paraphrases (Fuchs 1982): fondre comme neige au soleil $\rightarrow$ emagrecer do dia para a noite; n'écouter que d'une oreille $\rightarrow$ ouvir sem prestar atenção; quadrature du cercle $\rightarrow$ problema insolúvel, etc.

Il ne suffit point, pour le traducteur, d'être très conscient de la notion que les unités phraséologiques révèlent dans le texte de la langue étrangère, fait que l'on peut obtenir sans effort par l'intermédiaire des dictionnaires monolingues. La maîtrise des idiomatismes est indispensable au traducteur, non seulement parce qu'elle évite l'erreur fréquente de traduire littéralement les phraséologismes, mais aussi parce qu'elle permet de choisir parmi plusieurs synonymes celui qui stylistiquement s'approche le plus de l'original (Tristá 1988).

Alors, conscient de la difficulté de pouvoir compter sur une mémoire prodigieuse de toute son expérience culturelle et linguistique, au moment où il doit se souvenir, le plus vite possible, de correspondances précises, justement parce qu'il lui manque d'ouvrages de référence soigneux et plus complets, dans le secteur dictionnaires spéciaux, c'est le traducteur lui-même qui doit en premier déterminer les besoins de dictionnaires phraséologiques, qui pourraient correspondre à une importante économie d'efforts.

Les séries phraséologiques représentent, ainsi, un phénomène complexe et cohérent, dont le sens est régi par des mécanismes sémantiques profonds, mais qui attendent des recherches pour les élucider (Mejri 1994). Mais n'importe quel phraséologisme peut avoir, d'une langue à autre, un «traitement traductoire».

Claudia Maria Xatara Université Estadual Paulista, São José do Rio Preto, Brésil 


\section{RÉFÉRENCES}

BALly, C. (1951) : Traité de stylistique française, Paris, Klincksieck.

CARneado Moré, Z. V. (1985) : La fraseologia en los diccionarios cubanos, Havana Editorial de Ciencias Sociales.

EtTinger, S. (1982) : "Formación de palabras y fraseología en la lexicografía”, in G. HAEnsCH, et al. La lexicografía: de la lingüística teórica a la lexicografía práctica, Madrid, Gredos, pp. 233258.

Fuchs, C. (1982) : La paraphrase, Paris, PUF.

Galisson, R. (1988): "La culture partagée: une monnaie d'échange interculturelle», Lexiques, Paris, pp. 113-117.

MejRI, S. (1994): "Séquences figées et expression de l'intensité», Cahiers de lexicologie, 65, p. 111122.

Molinie, G. (1986) : Éléments de stylistique française, Paris, Presses Universitaires de France.

Rey, A. (1986) : "Les écarts culturels dans les dictionnaires bilingues ", Lexicographica, 2, p. 3342.

Rey, A. et S. Chantreau (1994) : Dictionnaire des expressions et locutions, $2^{\mathrm{e}}$ éd., Paris, Robert.

Rézeau, P. (1990): «Pour une étude des variantes géographiques et de la phraséologie du français", Cahiers de lexicologie, 56-1, p. 131-308.

Saussure, F. de (1969): Cours de linguistique générale, Paris, Payot.

TAGnin, S. E. O. (1988) : "A tradução dos idiomatismos culturais", Trabalhos de lingüística aplicada, 11, p. 43-52.

Tristá, M. A. (1988): Fraseología y contexto, Habana, Editorial de Ciencias Sociales.

Xatara, C. M. (1998): A tradução para o português das expressões idiomáticas em francês. Thèse de doctorat - UNESP, Araraquara.

\section{LA TRADUCCIÓN Y LA TERMINOLOCÍA EN LA COMUNICACIÓN BILINGÜE MEDIADA}

\section{Introducción}

La génesis de este artículo la constituyen los resultados de una investigación realizada bajo la modalidad de estudio de caso. El estudio se centró en un texto bilingüe, con el propósito de llegar a la comprensión de la situación traductiva que se sucede alrededor de él y poder formular conclusiones teóricas que permitan proponer un nuevo modelo de análisis donde se combine lo traductivo con lo terminológico desde la óptica de la teoría de la producción y organización del conocimiento científico en función del respectivo lenguaje profesional.

La primera aproximación a este libro permi- tió detectar una sintomatología en su constitución lingüístico-discursiva. Estos síntomas reveladores hicieron suponer la existencia de una patología que era necesario definir, al igual que sus causas. Se practicaron dos tipos de análisis (análisis traductivo y terminológico) cuyos resultados (inventario de errores) hicieron posible un diagnóstico (taxonomía de errores) sobre la patología que aquejaba al texto, sus causas y las acciones preventivas necesarias para evitar la recurrencia del mal.

Para su análisis, el libro es considerado integralmente, es decir, como un texto que intenta comunicar una misma información en dos lenguas a dos tipos de lectores cultural y lingüísticamente diferentes. El proceso investigativo aplicado al estudio particular de esta publicación permitió extrapolar unas conclu-siones teóricas sobre el deber ser del proceso traductivo, las competencias del traductor y la relación sine qua non entre traducción y terminología en la comunicación bilingüe mediada de lenguajes especializados.

\section{MEDELLIN \\ PANAMERICANOS \\ 2003 \\ PAN AMERICAN GAMES}

\author{
El Objeto de estudio: \\ un libro bilingüe del ámbito deportivo/ \\ institucional
}

La investigación eligió como objeto de estudio el libro bilingüe (español - inglés) "MEDELLIN, UNA CIUDAD PARA EL NUEVO MILENIO. JUEGOS PANAMERICANOS 2003"-MEDELLIN, A CITY FOR THE NEW MILLENNIUM. 2003 PAN AMERICAN GAMES”, elaborado con el fin de presentar a la ciudad de Medellín (sus características principales y la infraestructura deportiva disponible) como aspirante a sede de los Juegos Panamericanos del año 2003.

\section{Caracterización y ubicación del objeto de estudio según su campo temático y área de conocimiento.}

Como puede apreciarse a continuación en el Diagrama $\mathrm{N}^{\circ} 1$, el texto bilingüe objeto de estudio se ubica en el campo de los LENGUAJES ESPECIALIZADOS (Hoffmann, 1978) del área de conocimiento CIENCIAS DEL DEPORTE-eventos. El evento específico al que se refiere el texto son los JUEGOS PANAMERICANOS 2003, cuyas temáticas relacionadas tienen que ver con aspectos de la ciudad de MEDELLIN (hotelería, servicios médicos, ecología, escenarios, cultura, educación, festividades, ciudad deportiva) y LAS COMPETICIONES de dichos juegos (sedes, organización, deportes, alojamiento, escenarios). 